\title{
STATUT DE L'ÉCREVISSE À PATTES BLANCHES (AUSTROPOTAMOBIUS PALLIPES LEREBOULLET, 1858) EN BOURGOGNE : BILAN DE 5 ANNÉES DE PROSPECTION
}

\author{
D. LERAT (1), L. PARIS (2), P. BARAN (3)
}

(1) Société d'Histoire Naturelle d'Autun (SHNA), Observatoire de la Faune Patrimoniale de Bourgogne (OFAPB), Maison du Parc, 58230 St-Brisson, France.

(2) Parc naturel régional du Morvan (PNRM), Maison du Parc, 58230 St-Brisson, France.

(3) Conseil Supérieur de la Pêche (CSP), Délégation Régionale Bourgogne/FrancheComté, 22, boulevard du docteur Veillet, 21000 Dijon, France.

Reçu le 25 juillet 2005

Accepté le 12 janvier 2006

Received July 25, 2005

Accepted January 12, 2006

\section{RÉSUMÉ}

Bien que la répartition géographique actuelle de l'Écrevisse à pattes blanches soit un élément essentiel dans les politiques de gestion et de restauration de cette espèce, peu d'informations sont disponibles à l'échelle complète d'une région. En Bourgogne, depuis 2000 plusieurs organismes regroupés au sein du Groupe Écrevisse Bourguignon (GEB) ont engagé un programme régional commun de prospection. Après la vérification des données historiques, le travail, encore en cours, consiste en une prospection systématique du réseau hydrographique par observation nocturne à la lampe entre les mois de juin et d'octobre. 893 stations ont été échantillonnées appartenant à 593 cours d'eau. L'Écrevisse à pattes blanches est présente dans 128 ruisseaux. L'Écrevisse à pieds rouges (Astacus astacus) est présente dans 2 ruisseaux et 2 étangs. Quatre espèces allochtones ont été également recensées lors des prospections: Astacus leptodactylus, Orconectes limosus, Pacifastacus leniusculus, et Procambarus clarkii. La connaissance de la répartition à l'échelle régionale permet de mieux apprécier le statut de l'Écrevisse à pattes blanches. Elle n'est plus présente que dans la moitié des stations où elle était connue au cours des 30 dernières années. Elle est absente à présent des rivières larges et des zones de cultures ou fortement anthropisées. Seuls les ruisseaux de petite taille n'ayant pas subi d'altération physique, situés en forêt de feuillus et en prairie et pour lesquels la ripisylve est dense, sont encore colonisés. L'implantation d'étangs et l'enrésinement de bordure sont très défavorables à la présence d'une population d'écrevisse. Le statut de l'Écrevisse à pattes blanches en Bourgogne est donc très fragile. Les populations sont assez nombreuses mais extrêmement fragmentées et ne colonisent plus que des habitats refuges (ruisseaux situés en tête de bassin versant). La poursuite actuelle des politiques d'aménagement des milieux aquatiques (construction de plan d'eau, drainage des zones humides, enrésinement) et le très fort développement des écrevisses allochtones et plus particulièrement de l'Écrevisse de Californie menacent directement le maintien de l'Écrevisse à pattes blanches dans les bassins hydrographiques Bourguignons.

Mots-clés: Écrevisse à pattes blanches, Groupe Écrevisses Bourguignon, distribution régionale, statut, habitat actuel. 


\section{STATUS OF THE WHITE-CLAWED CRAYFISH (AUSTROPOTAMOBIUS PALLIPES LEREBOULLET, 1858) IN BURGUNDY : ASSESSMENT OF 5 YEARS OF SAMPLING}

\section{ABSTRACT}

At a regional scale, complete information about geographical distribution of the Whiteclawed crayfish is very rare, even if these data are the basic ones for suitable conservation and management programs. In Burgundy, since 2000, several organisms - gathered under the Burgundy Crayfish Group have decided to conduct a large regional program of crayfish distribution. After the validation of historical information, nocturnal sampling has been systematically conducted over all the watersheds between June and October. Since the beginning of this program, 893 stations have been sampled, from 593 streams. The Whiteclawed Crayfish have been found in 128 small rivers, the noble Crayfish (Astacus astacus) in only 2 streams and 2 ponds. Four non-indigenous species have also been recorded: Astacus leptodactylus, Orconectes limosus, Pacifastacus leniusculus, and Procambarus clarkii. Half of the stations - where the White-clawed Crayfish was recorded during the last 30 years - is no longer colonized. To date, stations colonized by this Heritage species correspond only to small rivers from of headwater with a dense riparian vegetation and running in hardwood forest or meadows. Concerning the stations under the effects of new ponds or with coniferous forest on the edge of the river, the White-clawed Crayfish is very rare. In Burgundy, the White-clawed Crayfish populations are not very rare but very isolated and consequently, the specie status is weak. The current policies about rivers and aquatic habitats management (new ponds, drainage of wetlands, increasing in coniferous forest surfaces...) and the invasion of Pacifastacus leniusculus directly, threaten the Whiteclawed crayfish populations in the Burgundy watersheds.

Key-words: White-clawed crayfish, Burgundy Crayfish Group, status, regional distribution, current habitat.

\section{INTRODUCTION}

Originellement, l'Écrevisse à pattes blanches (Austropotamobius pallipes) est une espèce largement répandue en Europe de l'Ouest avec une très grande diversité de milieux colonisés (VIGNEUX, 1997). Au cours du siècle dernier, cette espèce a connu une forte régression de son aire de distribution aboutissant à une fragilisation de son statut et une distribution très fragmentée des populations dans des zones marginales (ALONSO et al., 2000 ; LEGALLE et al., 2003). La mise en œuvre de politiques et d'actions de protection et de restauration est devenue totalement indispensable à la sauvegarde de l'espèce. Pour cela, l'appui de la connaissance notamment au niveau du statut constitue un préalable nécessaire. Dans ce domaine, outre les études de cas, la définition de la répartition des populations à des échelles larges (bassins, régions) à partir de prospections de terrain constitue une action déterminante pour la conservation de l'espèce.

En Bourgogne, il y a environ dix ans, le nombre de stations à écrevisses autochtones connues ne dépassait pas la dizaine. A partir de 1992, quelques populations d'Écrevisses à pattes blanches ont été redécouvertes dans le Morvan. Ces nouvelles données ont incité le Parc Naturel Régional du Morvan à prospecter dès 1994 les ruisseaux et rivières du massif (MAHIEU et PARIS, 1998). De son côté, le Conseil Supérieur de la Pêche au travers de ses brigades départementales avait également engagé un travail de prospection dans différents bassins (MARACHE, 2000). Ces premières observations ont confirmé la disparition de certaines populations et la fragilité du statut de l'espèce.

En 2000, différents partenaires (Conseil Supérieur de la Pêche, Parc Naturel Régional du Morvan, Direction Régionale de l'Environnement de Bourgogne, Agence de l'Eau Seine-Normandie, Fédérations Départementales pour la Pêche et la Protection 
du Milieu Aquatique, Société d'Histoire Naturelle d'Autun) se sont rassemblés pour organiser la collecte des informations sur les écrevisses à l'échelle de l'ensemble du territoire bourguignon. Rassemblés autour d'une structure informelle, le Groupe Écrevisse Bourguignon, les différents partenaires ont engagé un programme de travail visant:

- à standardiser la collecte des informations sur les populations d'écrevisses en Bourgogne,

- à organiser et coordonner des prospections,

- à réaliser une base de données pour la diffusion des informations, européens,

- à intégrer les données dans les zonages d'intérêt écologique bourguignons et

- à mettre en œuvre des actions de protection et/ou de restauration des habitats.

L'ensemble des informations recueillies depuis 2000 a été analysé avec pour objectif de fournir des données cartographiées sur le statut des écrevisses et notamment des écrevisses à pattes blanches en Bourgogne, d'analyser les évolutions récentes des populations de cette espèce ainsi que de caractériser les habitats actuellement colonisés.

\section{MATÉRIEL ET MÉTHODES}

\section{Les stations d'étude}

Le travail a été conduit à l'échelle complète de la région Bourgogne couvrant 4 départements. Cette région qui recoupe trois grands bassins hydrographiques (Seine, Loire, Saône) représente $64000 \mathrm{~km}$ de cours d'eau. Les prospections se sont centrées généralement sur des cours d'eau de faible largeur (inférieur à 3 mètres). La majorité des habitats prospectés se situent dans des environnements agricoles de type prairiaux ou forestiers a priori relativement préservés.

\section{Le plan d'échantillonnage : stratégie de prospection}

La récolte des données s'appuie sur deux types de prospections :

- des prospections spécifiques et ciblées pour une recherche d'écrevisses,

- des prospections non spécifiques conduites dans le cadre d'études générales, dans lesquelles la recherche de la faune aquatique patrimoniale est le plus souvent intégrée à présent ou bien de réseaux de suivi tel que le Réseau Hydrobiologique et Piscicole.

\section{Les prospections spécifiques}

Elles se sont appuyées sur deux niveaux d'approche:

- Le premier se base sur la vérification de données historiques. Ces données sont issues de nombreuses sources disponibles soit dans la littérature (ANDRE, 1960 ; DE DROUIN DE BOUVILLE, 1906 ; GEOFFROY SAINT-HILAIRE, 1884 ; LACHAT, 1985 ; LAURENT et LACHAT, 1988 ; LAURENT et SUSCILLON, 1962 ; MAHIEU et PARIS, 1998), soit au sein des différents organismes du Groupe Écrevisses Bourguignon notamment du Conseil Supérieur de la Pêche et des Fédérations Départementales pour la Pêche et la Protection du Milieu Aquatique.

- Le second repose sur une prospection ciblée dans des stations présentant la plus forte probabilité de présence d'écrevisses à pattes blanches. Ces prospections ont été engagées dans une deuxième phase après la vérification des données historiques. Cette probabilité est définie a priori à partir de critères environnementaux basés sur une faible pression anthropique, une zone géographique proche des sources et des habitats essentiellement forestiers et prairiaux. Cette approche a priori, non issue d'un plan d'échantillonnage systématique ou stratifié est complétée ponctuellement par une prospection de stations présentant de faibles potentialités d'accueil (habitat de grandes cultures, zones péri-urbaines...). 


\section{Les prospections non spécifiques}

Les données issues de prospections ponctuelles engagées dans le cadre d'étude de cours d'eau (COUASNE, 2003 ; BOUCHARD, 2004 ; ECOGEA, 2005 ; JOSSET, 2004) ou de réseau de suivi (Réseau Hydrobiologique et Piscicole) sont également retenues dans ce travail.

\section{Méthodes de récolte des données}

Dans le cadre des prospections spécifiques, les données sont issues d'observations nocturnes à la lampe. Sur chaque unité hydrographique sélectionnée, trois tronçons d'une longueur de $100 \mathrm{~m}$ au minimum sont choisis pour conduire la prospection. Ces tronçons sont le plus souvent positionnés à la source, à la première confluence avec un autre ruisseau et en position intermédiaire dans la zone délimitée entre la source et la confluence. Chaque tronçon est préalablement décrit de jour. Différentes caractéristiques de l'habitat et de l'environnement proche sont relevées sur le terrain (Tableau I). En complément, I'utilisation de base de données cartographiques CORINE Land Cover 2000 a permis de préciser les informations sur l'environnement immédiat des ruisseaux prospectés.

\section{Tableau I}

Descripteurs de l'habitat et de l'environnement de chaque station.

\section{Table I}

Descriptors of the habitat and the environment of each station.

\begin{tabular}{|l|l|l|}
\hline \multicolumn{1}{|c|}{ Descripteurs } & \multicolumn{1}{|c|}{ Descripteurs secondaires } & \multicolumn{1}{c|}{$\begin{array}{c}\text { Méthode } \\
\text { d'appréciation }\end{array}$} \\
\hline $\begin{array}{l}\text { Environnement proche du } \\
\text { cours d'eau }\end{array}$ & $\begin{array}{l}\text { Prairies, forêts résineuses, forêts } \\
\text { feuillues, zones humides,cultures, } \\
\text { zones urbaines, routes }\end{array}$ & Estimation en \% relatifs \\
\hline Présence d'étangs & $\begin{array}{l}\text { Sur le cours d'eau, en dérivation, } \\
\text { en amont, en aval }\end{array}$ & Estimation visuelle \\
\hline Occupation des berges & $\begin{array}{l}\text { Prairies, forêts résineuses, forêts } \\
\text { feuillues, zones humides,cultures, } \\
\text { zones urbaines, routes }\end{array}$ & Estimation en \% relatifs \\
\hline $\begin{array}{l}\text { Importance des chevelus } \\
\text { racinaires }\end{array}$ & & $\begin{array}{l}\text { Estimation en 4 classes } \\
\text { (fort, moyen, faible, nul) }\end{array}$ \\
\hline Ombrage & $\begin{array}{l}\text { Estimation en 4 classes } \\
\text { (fort, moyen, faible, nul) }\end{array}$ \\
\hline $\begin{array}{l}\text { Largeur moyenne de la } \\
\text { station }\end{array}$ & Mesure en mètres \\
\hline $\begin{array}{l}\text { Profondeur moyenne de } \\
\text { la station }\end{array}$ & & Mesure en mètres \\
\hline $\begin{array}{l}\text { Sinuosité au niveau de la } \\
\text { station }\end{array}$ & & $\begin{array}{l}\text { Estimation en 4 classes } \\
\text { (fort, moyen, faible, nul) }\end{array}$ \\
\hline Présence de sous-berges & & $\begin{array}{l}\text { Estimation en 3 classes } \\
\text { (nombreuses, peu } \\
\text { formées, nul) }\end{array}$ \\
\hline Vrai/faux \\
\hline $\begin{array}{l}\text { Travaux hydration de la qualité de } \\
\text { l'eau }\end{array}$ & $\begin{array}{l}\text { Élevage, cultures, domestiques, } \\
\text { industrielles, autres... }\end{array}$ & $\begin{array}{l}\text { Estimation visuelle. } \\
\text { Vrai/faux }\end{array}$ \\
\hline Granulométrie du fond & Huit classes de granulométrie & Estimation en \% relatifs \\
\hline Longueur de la station & & Mesure en mètres \\
\hline
\end{tabular}


De nuit, la prospection est conduite par deux opérateurs d'aval en amont. Chaque individu d'écrevisse est comptabilisé. Sans manipulation, la taille est estimée selon 6 classes (<30 mm, 30-49 mm, 50-69 mm, 70-89 mm, > $90 \mathrm{~mm}$ ) et le sexe est également déterminé sans manipulation en s'appuyant sur le dimorphisme sexuel secondaire (rapport de largeur entre abdomen et céphalothorax, taille des pinces), ceci pour les individus mâtures. Les mutilations éventuelles ou l'état sanitaire apparent sont également notés.

Les données des prospections non spécifiques sont issues de pêches électriques (appareil de type Héron Dream Electronic ou Martin-Pêcheur Dream Electronic). La méthode est basée sur les passages successifs (DE LURY, 1951).

Pour la majorité des stations, les prospections n'ont été réalisées qu'une seule fois.

\section{Stockage des données}

Les données recueillies dans des fiches de terrain standardisées sont stockées dans un système de gestion de base de données couplé à un Système d'Information Géographique.

\section{Traitement des données}

La variable biologique analysée correspond à la présence ou l'absence sur une station d'une espèce d'écrevisse. Cette variable a permis une description cartographique du statut des différentes espèces d'écrevisses rencontrées. Les données sont restituées par quadrat correspondant à 1 seizième de carte IGN série bleue au $1 / 25000^{\mathrm{e}}(0,05$ gra des $\times 0,05$ grades) pour un usage grand public, et en localisation précise pour un usage professionnel.

La présence ou l'absence de l'Écrevisse à pattes blanches a été confrontée aux caractéristiques de l'habitat et de l'environnement proche des différents ruisseaux. Pour chacune des modalités des différentes variables, le pourcentage de stations accueillant des écrevisses à pattes blanches a été calculé. Un test statistique du Khi2 (SPRENT, 1992) a été effectué afin de valider les différences entre les distributions.

\section{RÉSULTATS}

\section{Répartition des espèces d'écrevisses}

Depuis 2000, 893 stations correspondant à 593 cours d'eau ont été échantillonnés (Figure 1) pour un total de $350 \mathrm{~km}$ de petits cours d'eau prospectés. Conformément à la stratégie prospection, l'effort d'échantillonnage est concentré sur des secteurs géographiques bien particuliers (Morvan, Puisaye, Châtillonnais, Charollais, Brionnais, Auxois) présentant le plus de données historiques et les plus fortes potentialités d'accueil. Les zones de grandes cultures céréalières (plaine de Saône, Sénnonais, plateaux calcaires) ont été peu prospectées.

Six espèces d'écrevisses ont été observées sur les zones prospectées, l'Écrevisse à pattes blanches (Austropotamobius pallipes Lereboullet, 1858), l'Écrevisse à pieds rouges (Astacus astacus Linné, 1758), l'Écrevisse à pattes grêles (Astacus leptodactylus Eschscholtz, 1823), l'Écrevisse américaine (Orconectes limosus Rafinesque, 1817), l'Écrevisse de Californie (Pacifastacus leniusculus Dana, 1852) et l'Écrevisse de Louisiane (Procambarus clarkii Girard, 1852) (Tableau II).

Dans les échantillonnages réalisés, l'Écrevisse à pattes blanches reste la mieux représentée avec 200 stations appartenant à 128 cours d'eau (Figure 2) et représentant au total $50 \mathrm{~km}$ de cours d'eau colonisés. A l'opposé, l'Écrevisse à pattes grêles et l'Écrevisse de Louisiane sont peu abondantes (respectivement 8 et 6 stations pour les 2 espèces). 


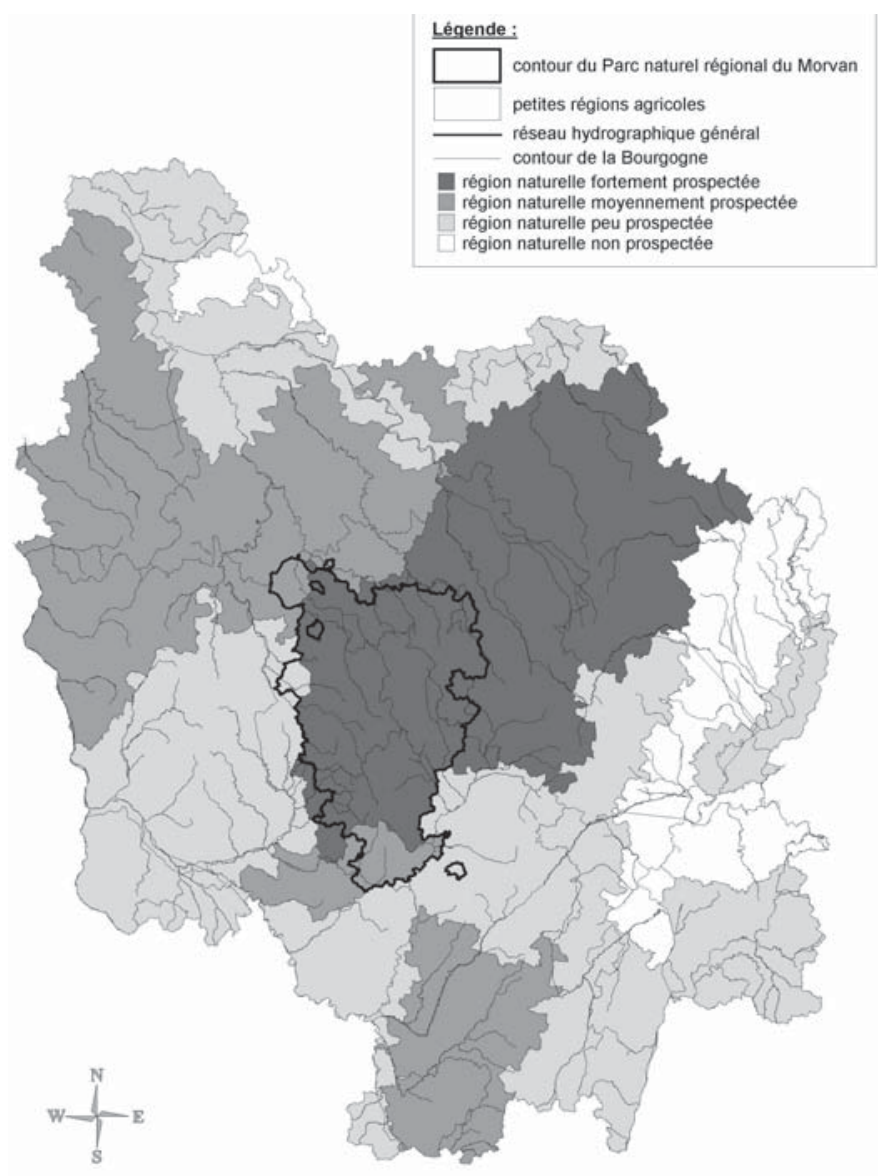

Echelle : $1 \mathrm{~cm}$ pour $236,6 \mathrm{Km}$

\section{Figure 1}

Relation entre l'effort de prospection par région naturelle et leur degré d'anthropisation.

\section{Figure 1}

Relation between sampling effort per natural region and the level of human influence.

\section{Tableau II}

Nombre de stations et de ruisseaux prospectées par espèce et par département en Bourgogne.

Table II

Number of sampled stations and per species and per department in Burgundy.

\begin{tabular}{|l|c|c|c|c|c|c|c|c|c|c|}
\cline { 2 - 10 } & \multicolumn{2}{|c|}{ Côte d'Or } & \multicolumn{2}{c|}{ Nièvre } & \multicolumn{2}{c|}{ Saône et Loire } & \multicolumn{3}{c|}{ Yonne } & \multicolumn{2}{c|}{ Total Bourgogne } \\
\cline { 2 - 11 } & $\begin{array}{c}\text { Nb de } \\
\text { stations }\end{array}$ & $\begin{array}{c}\text { Nb de cours } \\
\text { d'eau ou } \\
\text { étangs }\end{array}$ & $\begin{array}{c}\text { Nb de } \\
\text { stations }\end{array}$ & $\begin{array}{c}\text { Nb de cours } \\
\text { d'eau ou } \\
\text { étangs }\end{array}$ & $\begin{array}{c}\text { Nb de } \\
\text { stations }\end{array}$ & $\begin{array}{c}\text { Nb de cours } \\
\text { d'eau ou } \\
\text { étangs }\end{array}$ & $\begin{array}{c}\text { Nb de } \\
\text { stations }\end{array}$ & $\begin{array}{c}\text { Nb de cours } \\
\text { d'eau ou } \\
\text { étangs }\end{array}$ & $\begin{array}{c}\text { Nb de } \\
\text { stations }\end{array}$ & $\begin{array}{c}\text { Nb de cours } \\
\text { d'eau ou étangs }\end{array}$ \\
\hline Austropotamobius pallipes & 30 & 20 & 89 & 53 & 53 & 45 & 28 & 10 & 200 & 128 \\
\hline Astacus astacus & 0 & 0 & 6 & 3 & 0 & 0 & 1 & 1 & 7 & 4 \\
\hline Pacifastacus leniusculus & 19 & 6 & 29 & 18 & 9 & 5 & 8 & 5 & 65 & 34 \\
\hline Orconectes limosus & 19 & 14 & 14 & 12 & 4 & 4 & 15 & 12 & 52 & 42 \\
\hline Astacus leptodactylus & 0 & 0 & 6 & 5 & 2 & 1 & 0 & 0 & 8 & 6 \\
\hline Procambarus clarkii & 1 & 1 & 1 & 1 & 1 & 1 & 3 & 3 & 6 & 6 \\
\hline Aucune & 173 & 128 & 161 & 112 & 83 & 61 & 138 & 72 & 555 & 373 \\
\hline
\end{tabular}




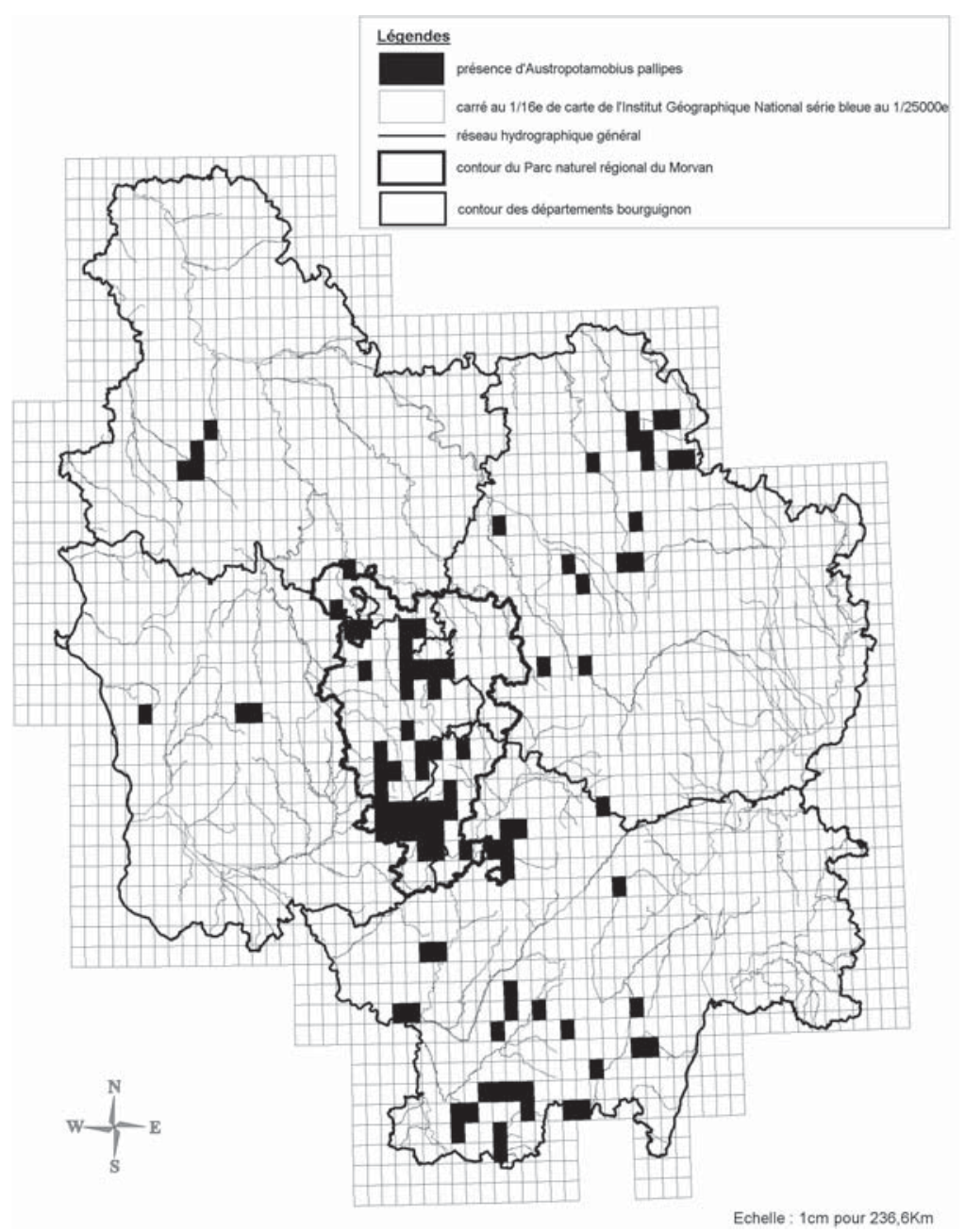

Figure 2

Répartition d'Austropotamobius pallipes en Bourgogne.

Figure 2

Distribution of the White-clawed crayfish in Burgundy.

En ce qui concerne les écrevisses nord-américaines, le nombre de stations est certainement très sous-évalué compte tenu du type de cours d'eau prospectés. Les chiffres indiquent plutôt un nombre de stations où potentiellement nous aurions pu redécouvrir l'Écrevisse à pattes blanches. Cette remarque est surtout vraie pour l'Écrevisse de Californie qui semble affectionner particulièrement des habitats lotiques équivalents à ceux occupés par l'Écrevisse à pattes blanches.

Jusqu'à présent, la connaissance de la répartition en Bourgogne des espèces invasives n'est pas l'objectif du travail réalisé par le Groupe Écrevisses Bourguignon. Les données stockées dans l'Astabase, si elles donnent une image fidèle a priori de la répartition de l'Écrevisse à pattes blanches, ne permettent pas une cartographie et une analyse pertinente sur les espèces nord-américaines.

L'Écrevisse à pattes blanches n'occupe plus de grands linéaires mais plutôt des petits secteurs fragmentés de quelques dizaines à quelques centaines de mètres. Les 
densités se situent dans une fourchette allant de 1 individu/100 $\mathrm{m}^{2}$ à 237 individus/100 $\mathrm{m}^{2}$. Dans $40 \%$ des cas la densité ne dépasse pas 10 individus $/ 100 \mathrm{~m}^{2}$.

Lors de nos prospections, un peu plus de 7000 individus ont été dénombrés. Ce chiffre est le reflet que d'une valeur d'échantillonnage et non une indication du stock d'écrevisses à pattes blanches en Bourgogne. Depuis 2000, l'effort annuel de prospection s'est toujours traduit par la découverte de nouvelles stations. En moyenne, 18 nouvelles stations à Écrevisse à pattes blanches sur 100 nouvelles stations prospectées sont découvertes chaque année (Figure 3). Ainsi, de nouvelles populations restent probablement à localiser, même si l'on sait que les milieux potentiels pour l'espèce se raréfient.

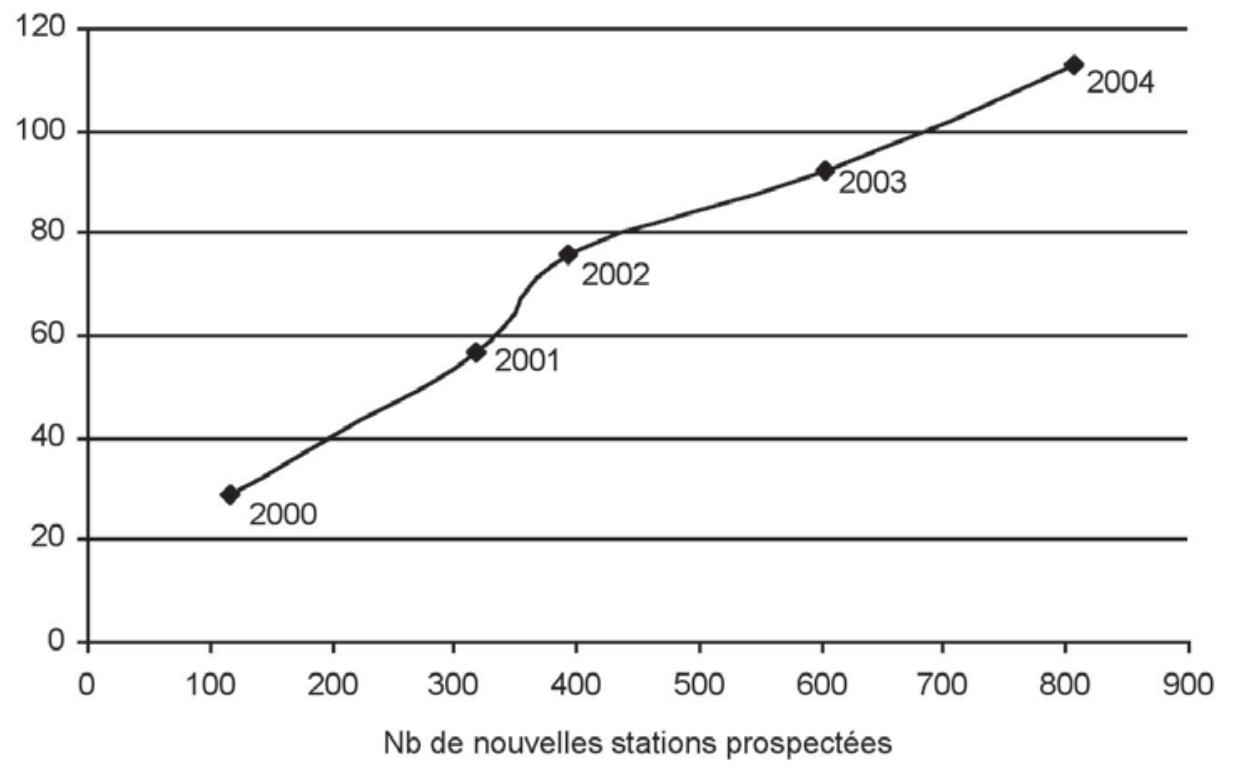

Figure 3

Courbe de ratio entre le $\mathrm{Nb}$ de nouvelles stations à Austropotamobius pallipes et le $\mathbf{N b}$ de nouvelles stations prospectées de 2000 à 2004.

\section{Figure 3}

Curve of ratio between the number of new stations of Austropotamobius pallipes and the number of new sampled stations of 2000 to 2004.

\section{Évolution du nombre de populations}

En 2000, des informations historiques sur la présence d'écrevisses à pattes blanches avaient été recensées sur 132 stations. La majorité des informations (60,6\%) étaient postérieures à 1990 et issues des premières prospections effectuées sur le territoire du Morvan. Sur les 132 stations, seules 66 populations soit $50 \%$ des stations ont été retrouvées. Plus les données historiques sont anciennes et plus la probabilité de disparition de la station est forte. Pour les stations connues avant 1980 , seuls $26,7 \%$ accueillent encore des écrevisses après 2000. A l'opposé, pour celles connues depuis $1990,63,7 \%$ ont été retrouvées. Depuis le début du suivi en 2000, $19 \%$ des stations où l'espèce avait été observée ont disparu.

\section{Caractérisation de l'habitat actuel de l'Écrevisse à pattes blanches}

La largeur des ruisseaux influence la présence de l'espèce en Bourgogne (Figure 4). L'Écrevisse à pattes blanches est significativement plus représentée dans les ruisseaux 
dont la largeur est comprise entre 0,5 et $1 \mathrm{~m}$ de large (test du Khi2, $\mathrm{p}<0.05$ ). Aucune population n'a été observée récemment sur des rivières de plus de 3 mètres de large.

Les limites aval des populations connues sont proches des sources du cours d'eau. $52 \%$ d'entre elles sont à moins de $1 \mathrm{~km}$ des sources et $81 \%$ sont à moins de $2 \mathrm{~km}$ (dans une fourchette allant de $10 \mathrm{~m}$ à $6 \mathrm{~km}$ ).

Les populations d'écrevisses à pattes blanches sont très majoritairement présentes dans des environnements proches de type prairie et forêt (Tableau III). Seules $4 \%$ des stations sont situées en zone de cultures céréalières intensives. Aucune observation n'a été effectuée dans les zones urbanisées. Le croisement avec les informations issues de CORINE Land Cover confirme les données issues des observations de terrain avec $91 \%$ des stations à écrevisses à pattes blanches situées en zone dominée par la prairie ou la forêt (test effectué sur 155 stations où l'Écrevisse à pattes blanches est présente).

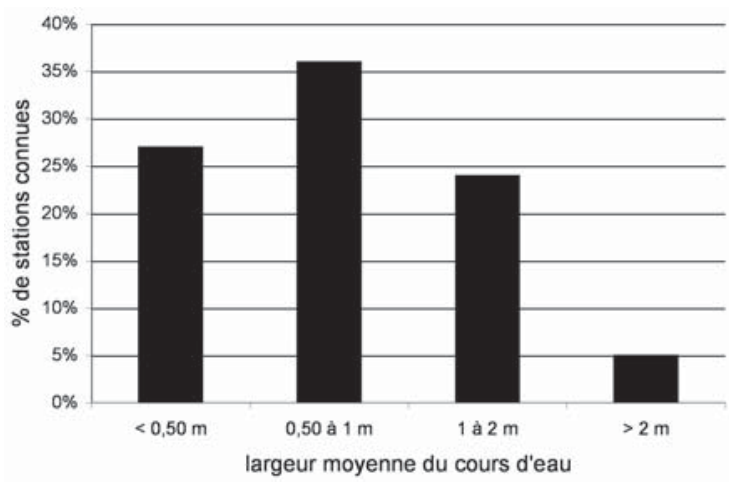

Figure 4

Relation entre le pourcentage de stations hébergeant Austropotamobius pallipes et la largeur moyenne des cours d'eau.

Figure 4

Percentage of stations with Austropotamobius pallipes compared with the average width of the stream.

\section{Tableau III}

Pourcentage de stations hébergeant Austropotamobius pallipes croisé avec la couche d'occupation des sols obtenus avec CORINE Land Cover.

Table III

Known stations with Austropotamobius pallipes, crossed with soil occupation obtained under CORINE Land Cover Information system.

\begin{tabular}{|l|c|}
\hline \multicolumn{1}{|c|}{ Nomenclature CORINE Land Cover } & $\begin{array}{c}\text { Pourcentage de stations à } \\
\text { Austropotamobius pallipes }\end{array}$ \\
\hline Forêts de conifères & $9 \%$ \\
\hline Forêts de feuillus & $39,3 \%$ \\
\hline Forêts mélangées & $13 \%$ \\
\hline Prairies & $29,7 \%$ \\
\hline Systèmes culturaux et parcellaires complexes & $0,6 \%$ \\
\hline Terres arables hors périmètres d'irrigation & $2,6 \%$ \\
\hline Territoires principalement occupés par l'agriculture & $5,8 \%$ \\
\hline
\end{tabular}


La présence et la densité de la ripisylve influencent significativement la distribution des écrevisses à pattes blanches dans nos échantillonnages. Le pourcentage de stations accueillant l'espèce est significativement plus élevé lorsque la ripisylve est dense $(31,4 \%)$ que lorsqu'elle est absente $(14,8 \%)$ (test du Khi2, p < 0.05) (Figure 5).

De même, l'état des abris en sous-berge joue un rôle vis-à-vis de la présence des écrevisses à pattes blanches. Le pourcentage de stations où l'espèce est recensée est significativement plus élevé dans les tronçons où les sous-berges sont bien formées $(40 \%)$ que dans ceux où elles sont peu formées $(20 \%)$ ou absente $(10 \%)$ (test du Khi2, $\mathrm{p}<0.05$ ) (Figure 6).

A habitat équivalent (station à ripisylve boisée et à sous-berges formées) et dans des environnements proches similaires (zones de prairies ou de forêts), aucune différence de présence d'écrevisses à pattes blanches n'a pu être statistiquement mis en évidence entre les bassins hydrographiques de la Seine et de la Loire.

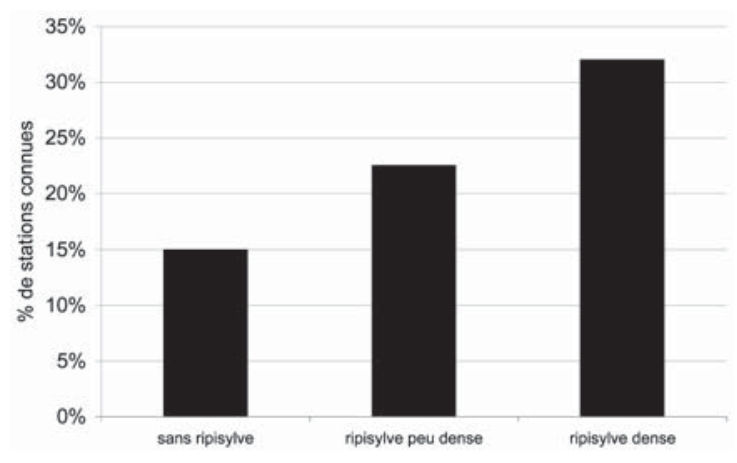

Figure 5

Relation entre le pourcentage de stations connues à Austropotamobius pallipes et l'état de la ripisylve.

\section{Figure 5}

Percentage of stations with Austropotamobius pallipes compared with the state of ripirian forest.

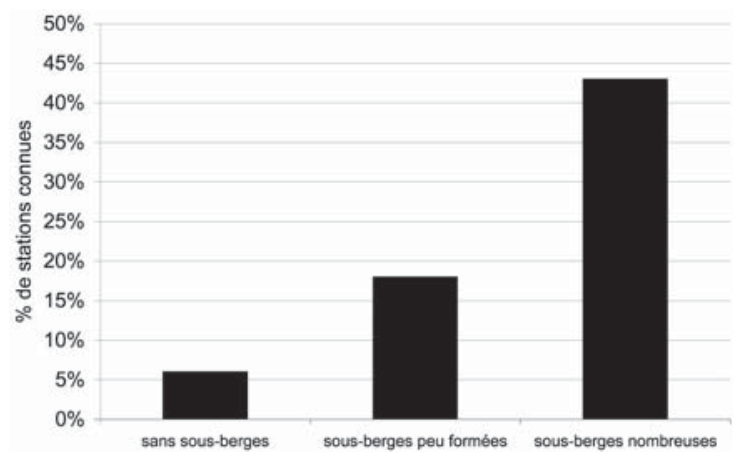

Figure 6

Relation entre le pourcentage de stations connues à Austropotamobius pallipes et l'état des sous-berges.

\section{Figure 6}

Percentage of stations with Austropotamobius pallipes compared with the importance of the sub-banks. 


\section{DISCUSSION}

L'approche conduite au sein du Groupe Écrevisse Bourguignon basée sur un très fort travail de prospection a permis d'apporter de nombreuses informations sur le statut des écrevisses et notamment de l'Écrevisse à pattes blanches à l'échelle complète d'une région: la Bourgogne. De nombreux travaux de ce type existent en Europe, mais ils sont essentiellement basés sur une approche à l'échelle des territoires nationaux (ALMAçA, 1989 ; ALONSO et al., 2000 ; CHANGEUX, 2003). L'approche au niveau d'une région française permet une analyse plus fine à l'échelle des sous-unités hydrographiques.

\section{Habitat, régression et cause de raréfaction de l'Écrevisse à pattes blanches en Bourgogne}

Actuellement, en Bourgogne, les populations d'écrevisses à pattes blanches sont fragmentées et de petites tailles. Elles trouvent majoritairement refuge dans des cours d'eau caractérisés par de faibles dimensions (entre 0,5 et 1 mètre de large) à proximité des sources dans des habitats peu altérés (ripisylve dense et sous-berges bien formées). Ces informations confirment les premières analyses effectuées antérieurement (MAHIEU et PARIS, 1998 ; CROQUET, 2003).

Cette situation est similaire à celle décrite dans d'autres pays. En Espagne, ALONSO et al. (2000) signalent des populations très fragmentées et subsistant dans des zones marginales. Au Portugal, ALMAÇA (1989) ou en Grande-Bretagne SMITH et al. (1996) indiquent également un statut très fragile pour les populations d'écrevisses à pattes blanches.

Ce constat découle majoritairement d'une forte régression de l'aire de distribution de l'espèce. Le travail conduit depuis 2000 a confirmé cela dans le réseau hydrographique bourguignon avec une perte de l'ordre de $50 \%$ des stations connues. Cette observation régionale est similaire à celles effectuées au niveau national lors de l'enquête de CHANGEUX (2003) qui indique une forte régression des espèces autochtones. Déjà en 1993, lors des premiers travaux dans le Morvan MAHIEU et PARIS (1998) mentionnaient des disparitions importantes de populations par rapport aux données du début du siècle. Cette disparition est également confirmée dans d'autres pays européens (ALONSO et al., 2000 ; CUELLAR et CUELLAR, 2000).

De nombreux travaux font état en France des disparitions massives des écrevisses autochtones suite à des épidémies de peste des écrevisses au cours du XXe siècle. En Bourgogne on cite des disparitions dans les années 1920, 1950 et 1970 (LAURENT et LACHAT, 1988).

La régression récente constatée dans nos travaux est un phénomène continu au cours des 30 dernières années, phénomène dans lequel les épizooties n'ont pas été identifiées comme la cause première (PARIS, 1999). II faut toutefois indiquer l'hétérogénéité des informations historiques disponibles notamment celles d'avant 1990, données issues d'archives ou de témoignages et non de prospections systématiques ce qui leur confère une incertitude. En revanche depuis 1990 et notamment dans le territoire du Parc naturel régional du Morvan, les données sont cartographiées et décrites de façon précises. La disparition de $40 \%$ des stations recensées à cette date est donc une information totalement fiable quant à la régression de l'espèce.

L'analyse de la répartition actuelle de l'espèce sur le territoire bourguignon apporte des informations importantes quant aux habitats encore utilisés par celle-ci ainsi qu'aux causes potentielles de sa disparition. Les stations d'écrevisses à pattes blanches ne sont plus présentes dans la totalité de leur habitat originel mais dans des zones refuges situées à proximité des sources. Dans un travail détaillé sur le pays Basque GARCIA-ARBERAS 
et RALLO (2000) ont identifié des populations d'écrevisses à pattes blanches sur des tronçons très réduits dans des ruisseaux de petites tailles.

Dans ces milieux aquatiques, les écrevisses sont présentes à la fois dans des environnements entièrement forestiers mais également prairiaux. Ces zones sont bien colonisées dès l'instant où les berges ne sont pas destructurées par le piétinement des animaux. Dans la première analyse conduite sur le territoire du Morvan, MAHIEU et PARIS (1998) signalent que $66 \%$ des populations sont présentes dans les zones de prairies.

La stabilité et la diversité des berges jouent un rôle déterminant vis-à-vis de la présence des écrevisses dans les ruisseaux de Bourgogne. Par leurs systèmes racinaires, la végétation rivulaire qu'elle soit ligneuse ou herbacée offre des habitats d'abris et de refuge indispensables aux écrevisses quelque soient leurs tailles.

L'espèce possède des préférences marquées pour certaines caractéristiques de I'habitat (DAGUERRE DE HUREAUX et ROQUEPLO, 1981). De nombreux auteurs signalent une forte relation entre l'importance et la diversité du couvert végétale des berges et l'abondance des écrevisses (FOSTER, 1995 ; SMITH et al., 1996 ; LACHAT et LAURENT, 1988 ; ROQUEPLO, 1997 ; NAURA et ROBINSON, 1998 ; REYJOL et ROQUEPLO, 2002).

Globalement, les ruisseaux à Écrevisses à pattes blanches en Bourgogne sont des habitats à forte diversité du point de vue des berges, de la granulométrie des fonds et des faciès d'écoulement. Dans une étude de deux ruisseaux du Morvan, ROUBEAU (1994) montre clairement les relations entre la répartition des écrevisses observées de nuit et la diversité des habitats, qu'ils soient liés à la granulométrie ou à la végétation.

L'analyse des données recueillies indique que l'espèce est systématiquement absente des secteurs ayant subi des altérations récentes de leur morphologie (curage, rectification, agriculture et sylviculture intensive, urbanisation, enrésinement, débardage, création d'étang, drainage des zones humides...) (LEGALLE et al., 2003).

\section{Limites de la méthode}

La méthode échantillonne un linéaire de cours d'eau, et ne donne donc pas une image complète des populations astacicoles. L'objectif est pourtant à terme de réaliser les inventaires sur les linéaires complets. Les moyens humains à mettre en place sont toutefois très importants.

Les prospections effectuées excluent les stations où les impacts anthropiques sont physiquement visibles. Aucune analyse fiable concernant la relation entre impacts anthropiques et présence d'écrevisses à pattes blanches ne peut être extraite de la base de données, tant que les linéaires complets des cours d'eau n'auront pas été échantillonnés et décrits.

Les informations recueillies ne permettent pas de mettre en évidence les effets d'altérations de la qualité des eaux.

Le travail réalisé cible particulièrement une espèce, l'Écrevisse à pattes blanches. Le type de rivières prospectées (têtes de bassin) ne permet pas d'avoir un inventaire objectif des espèces allochtones. En revanche, la méthode semble appropriée pour l'Écrevisse à pieds rouges, car les seules stations connues à ce jour en Bourgogne sont issues de nos prospections.

\section{CONCLUSION}

Le bilan des 5 années de prospections axées sur la connaissance de l'Écrevisse à pattes blanches en Bourgogne met en avant que les efforts de prospections 
systématiques sont fructueux puisqu'ils ont permis de retrouver 128 cours d'eau où l'espèce est encore présente, ce qui est au-delà de nos espérances. Par contre, les faibles densités et les linéaires colonisés de faible longueur sont des constats parfois inquiétants pour le devenir des populations en Bourgogne. Ces populations sont d'autant plus fragiles qu'elles se cantonnent maintenant sur des cours d'eau de faible dimension.

Les informations acquises et stockées dans une base de données cartographique permettent de mieux cerner les exigences en terme d'habitat des populations d'Écrevisse à pattes blanches encore en place. Elles permettent aussi d'envisager des opérations de reconquête de milieux ciblés sur des bassins encore naturellement occupés. Le porté à connaissance de ces données, la communication sont aussi des actions indispensables, pour éviter des disparitions parfois dues à l'ignorance. Le Programme LIFE NATURE "Ruisseaux de têtes de bassins et faune patrimoniale associée » piloté par le Parc naturel régional du Morvan pour 4 ans apportera des réponses, nous l'espérons, sur les possibilités de maintien et de propagation des populations d'écrevisses à pattes blanches encore présentes en Bourgogne.

L'inventaire est loin d'être complet en Bourgogne.

Les découvertes de nouveaux sites se font de plus en plus rares, mais pourtant l'importance du réseau hydrographique bourguignon nous laisse encore espérer la découverte de quelques populations supplémentaires d'écrevisses à pattes blanches. L'effort de prospection doit se poursuivre. En revanche la probabilité de trouver des populations de plusieurs milliers d'individus sur des linéaires importants comme celles décrites en Bourgogne par le passé semble nulle.

La connaissance de ces populations relictuelles, la préservation du milieu et de la qualité de l'eau sont indispensables pour maintenir l'espèce et envisager leur propagation, naturelle ou aidée, vers une reconquête à large échelle des cours d'eau.

\section{REMERCIEMENTS}

Les auteurs tiennent à remercier pour leur collaboration active notamment dans le domaine des prospections l'ensemble des agents des 4 brigades départementales du Conseil Supérieur de la Pêche de Bourgogne, les 4 Fédérations Départementales des Associations de Pêche et de Protection du Milieu Aquatique de Bourgogne, le Parc naturel régional du Morvan, le LIFE NATURE "Ruisseaux de têtes de bassins et faune patrimoniale associée ", la Société d'Histoire Naturelle d'Autun, tous les observateurs sans qui ce travail ne pourrait exister et les services de l'état et de la région pour l'intérêt qu'ils portent aux inventaires naturalistes.

Ce travail s'intègre dans l'Observatoire de la Faune Patrimoniale de Bourgogne qui est un programme de la Société d'Histoire Naturelle d'Autun financé par l'Agence de l'Eau Seine-Normandie, le Conseil Régional de Bourgogne, la Direction Régionale de l'Environnement de Bourgogne et par des fonds européens.

\section{BIBLIOGRAPHIE}

ALMAÇA C., 1989. On the biology of north-eastern Portuguese populations of Austropotamobius pallipes (Lereboullet, 1858). Arch. Museu Bocage, nov. Ser., I, $29,419-428$.

ALONSO F., TEMINO C., DIEGUEZ-URIBEONDO J., 2000. Status of the White-clawed crayfish, Austropotamobius pallipes (Lereboullet, 1858), in Spain : distribution and legislation. Les espèces natives d'Europe, Bull. Fr. Pêche Pisci., 356, 31-54. 
ANDRE M., 1960. Les écrevisses françaises. Éd. P. LECHEVALIER, Paris, 293 p.

BOUCHARD J., 2004. Potentialités des affluents de l'Ouche en amont de Dijon vis-à-vis de la reproduction de la truite commune (Salmo trutta L.), Mémoire de stage, DESS Ingénierie des Hydrosystèmes Continentaux en Europe. Conseil Supérieur de la Pêche, Université de Tours, $137 \mathrm{p}$.

CHANGEUX T., 2003. Évolution de la répartition des écrevisses en France métropolitaine selon les enquêtes nationales menées par le Conseil Supérieur de la Pêche de 1977 à 2001. Les écrevisses européennes autochtones en tant qu'espèce clef, reliant science, gestion et économie à un environnement durable. In : REYNOLDS J., SOUTY-GROSSET C. eds. : The endangered native crayfish Austropotamobius pallipes, Bioindicator and heritage species, CRAYNET, volume 1, Bull. Fr. Pêche Piscic., 370-371, 17-37.

CORINE LAND COVER, 2000. BD Carto@ de l'Institut Géographique National. Couches occupation des sols et réseaux. Institut Français de l'Environnement.

COUASNE J.P., 2003. Restauration de la libre circulation piscicole sur les affluents salmonicoles de la Cure entre les réservoirs des Settons et du Crescent, Mémoire de stage, DESS Ingénierie des Hydrosystèmes Continentaux en Europe. Parc naturel régional du Morvan \& Fédération Départementale des Associations de Pêche et de la Protection des Milieux Aquatiques de la Nièvre, Université de Tours, $126 \mathrm{p}$.

CROQUET V., 2003. Analyse des facteurs environnementaux influençant la répartition de l'Écrevisse à pattes blanches en Bourgogne, Mémoire de stage, DESS Espace Rural et Environnement. Conseil Supérieur de la Pêche, Délégation Régionale Bourgogne/Franche-Comté, $42 \mathrm{p}$.

CUELLAR L., CUELLAR M.C., 2000. Évolution de l'aphanomycose et repeuplements avec l'Écrevisse signal (Pacifastacus leniusculus) en Espagne. L'astaciculteur de France, 65, 2-9.

DAGUERRE DE HUREAUX N., ROQUEPLO C., 1981. Définition du biotope préférentiel de l'Écrevisse à pattes blanches, Austropotamobius Pallipes (Ler.) dans un ruisseau landais. Bull. Fr. Pêche Piscic., 281, 211-222.

DE DROUIN DE BOUVILLE R., 1906. Les repeuplements en écrevisses. Éd. Berger LEVRAULT, Paris, Nancy, $113 \mathrm{p}$.

DE LURY D.B., 1951. On the planning of experiments for the estimation of fish populations. J. Fish. Reds. Bd. Can., 18 (4), 281-307.

ECOGEA., 2005. Reconnections de cours d'eau - Étude piscicole et hydrologique sur les bassins-versants des Ruisseaux du Moulin Granard et de l'Abeille en vue de l'amélioration de la circulation piscicole, 71 p. plus annexe.

FOSTER J., 1995. Factors influencing the distribution and abundance of the crayfish Austropotamobius Pallipes in Wales and the Marches, UK. Freshwater Crayfish, VIII, 79-98.

GARCIA-ARBERAS L., RALLO A., 2000. Survival of natural populations of Austropotamobius Pallipes in rivers in Bizkaia, Basque Country (North of Iberian Peninsula). Les espèces natives d'Europe, Bull. Fr. Pêche Piscic., 356, 17-30.

GEOFFROY SAINT-HILAIRE E., 1884. Procès verbal de séance. Bull. de la Sté Zool. d'Acclimatation, $508 \mathrm{p}$.

JOSSET A., 2004. Monographie d'une tête de bassin à Écrevisse à pattes blanches dans le Morvan, Mémoire de stage, DESS Qualité et Traitement de l'Eau et Bassin Versant. Parc naturel régional du Morvan, $53 \mathrm{p}$. 
LACHAT G., 1985. L'écrevisse en Morvan, Mémoire de fin d'études ENITA de DijonQuétigny, $112 \mathrm{p}$.

LACHAT G., LAURENT P.J., 1988. The habitats of Astacus astacus L. and Austropotamobius Pallipes LERE. In the Morvan. Freshwater crayfish, 7, 61-68.

LAURENT P.J., LACHAT G., 1988. Les écrevisses du Morvan, hier, aujourd'hui, demain. Image de la Saône-et-Loire, 74, 3-7.

LAURENT P.J., SUSCILLON M., 1962. Les écrevisses en France. Annales de la Station Centrale d'Hydrobiologie appliquée, 9, 336-395.

LEGALLE M., CEREGHINO R., MASTROTILLO S., 2003. Conservation des populations aquatiques menacées : exemple de l'Écrevisse à pattes blanches, du chabot et de la moule perlière sur le bassin du Célé. Revue de l'Agence de l'Eau, 86, 10-16.

MAHIEU J., PARIS L., 1998. Les écrevisses en Morvan. Cahiers scientifiques. Parc naturel régional du Morvan, 1, $68 \mathrm{p}$.

MARACHE G., 2000. Atlas des écrevisses en Côte d'Or. Rapport Conseil Supérieur de la Pêche de la Côte d'Or, 17 p.

NAURA M., ROBINSON M., 1998. Principles of using River Habitat Survey to predict the distribution of aquatic species: an example applied to the native White-clawed crayfish Austropotamobius pallipes. Aquatic Conserv. Mar. Freshw. Ecosyst., 8, 515-527.

PARIS L., 1999. La raréfaction des écrevisses autochtones en Morvan. Conséquences de l'évolution de l'occupation du sol entre 1950 et 1993. Parc naturel régional du Morvan, $7 \mathrm{p}$.

REYJOL Y., ROQUEPLO C. 2002. Preferential habitat analysis of the White-clawed crayfish, notably juveniles, in the three brooks of Corrèze, France (F). In : SOUTYGROSSET C. and GRANDJEAN F. : Knowledge-based management of European native crayfishes, Crayfish special, volume 4, Bull. Fr. Pêche Pisci., 367, 741-759.

ROQUEPLO C., 1997. Étude de populations d'écrevisses à pattes blanches (Austropotamobius Pallipes Ler.) en cours d'eau, soumises à une pêche de loisir. L'Astaciculteur de France, 51, 59-68.

ROUBEAU P., 1994. Essai de caractérisation du biotope de l'Écrevisse à pattes blanches (Austropotamobius pallipes) dans deux ruisseaux du Parc naturel régional du Morvan, Mémoire de stage, Maîtrise BED. Parc naturel régional du Morvan, $81 \mathrm{p}$.

SMITH G.R.T., LEARNER M.A., SLATER F.M., FOSTER J., 1996. Habitat features important for the conservation of the native crayfish Austropotamobius Pallipes in Britain. Biol. Conserv., 75, 239-246.

SPRENT P., 1992. Pratique des statistiques non-paramétriques. Institut National de la Recherche Agronomique, Paris, $294 \mathrm{p}$.

VIGNEUX E., 1997. Les introductions de crustacés décapodes d'eau douce en France. Peut-on parler de gestion ? Bull. Fr. Pêche Piscic., 344-345, 357-370. 
